\title{
Simulated Micro-Electrode Array Recordings From Stem Cell-Derived Cardiomyocytes
}

\author{
Louise A. Bowler ${ }^{1}$, Kate Harris ${ }^{2}$, David J. Gavaghan ${ }^{1}$ and Gary R. Mirams ${ }^{1}$
}

${ }^{1}$ Computational Biology Group, Department of Computer Science, University of Oxford

${ }^{2}$ Safety Assessment, GlaxoSmithKline, Ware, UK

\section{Introduction}

Testing a novel pharmaceutical compound for adverse cardiac side-effects is a vitally important stage of the drug discovery process.

Human induced pluripotent stem cell-derived cardiomyocytes (hiPSC-CMs) are a promising cell type for cardiac safety testing, but differences between these cells and those in the adult heart mean that results must be interpreted with care.

The micro-electrode array (MEA) is a particularly interesting safety test because it demonstrates how tissue, rather than a single cell, responds to the presence of a compound

\section{Can models of the micro-electrode array help interpret the experimental results?}

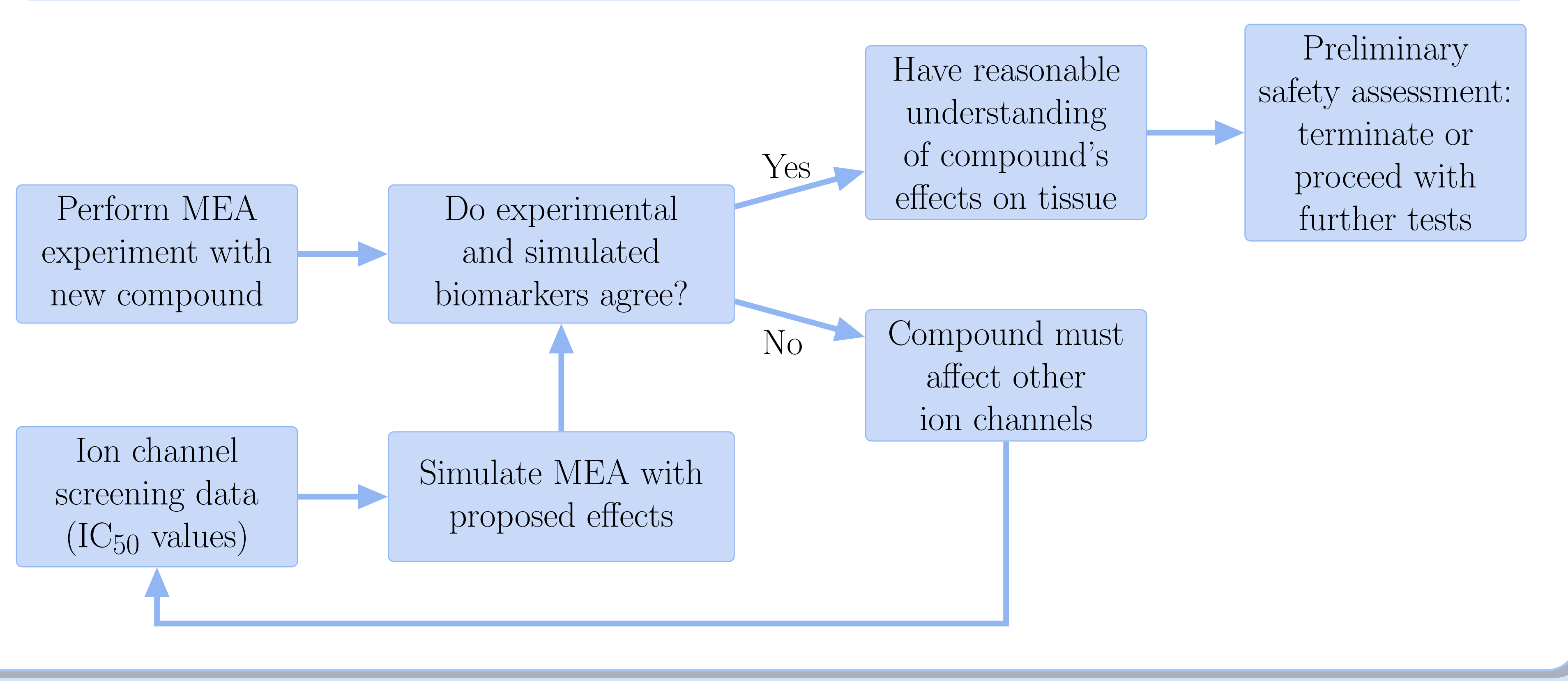

\section{Simulation Design}

\section{Simulating the Field Potential}

- The bidomain equations describe how the membrane and extracellular potentials vary over cardiac tissue. The equations were solved using Chaste, a $\mathrm{C}++$ library with a finite element solver for cardiac electrophysiology.

- The ionic current was calculated using a cellular electrophysiology model: in this case the atrial-like and ventricular-like hiPSC-CM models of Paci et al. (2013).

- Biomarkers of interest such as the beat rate and field potential duration were derived rom the simulated extracellular potential.

\section{Convergence Analysis}

- A square mesh (the same diameter of the MEA) was created with regularly-spaced nodes.

- The space between the nodes and the time-steps used to solve the system were varied.

- Space and time-steps were chosen so that reducing either of them further had no appreciable effect on the field potential or the values of the biomarkers
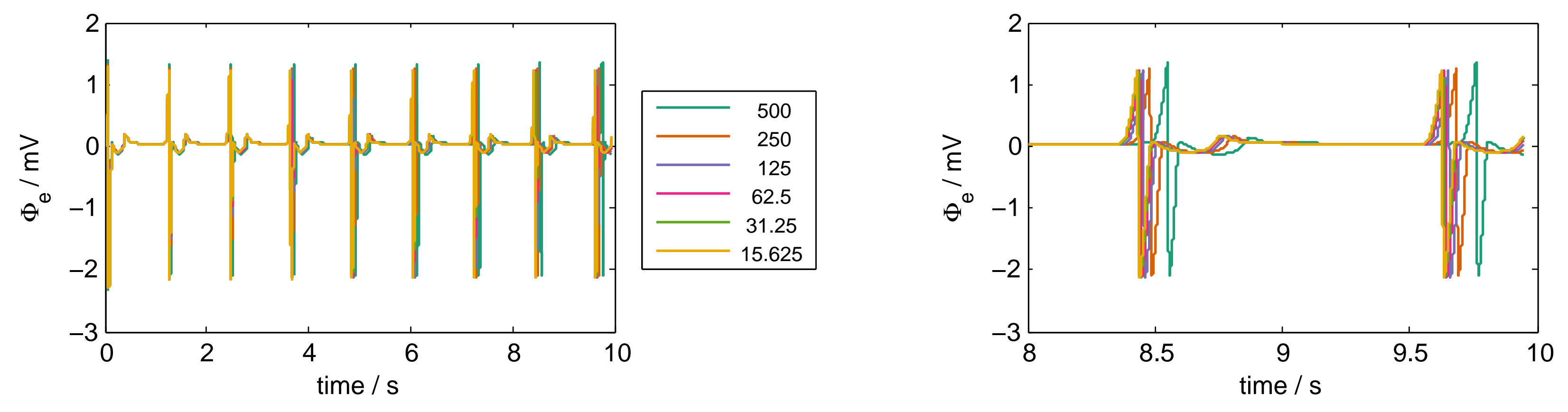

Above: Extracellular potential from simulations using different values of inter-node spacing (in $\mu \mathrm{m}$ )

\section{MEA Simulations}

- A 2D mesh was generated to model the monolayer of cells within the MEA, with maximum inter-node spacing of $150 \mu \mathrm{m}$. The time-step was $0.05 \mathrm{~ms}$.

- 20s of activity was simulated at each drug concentration.

- The extracellular potential was recorded at the locations of the 60 electrodes.
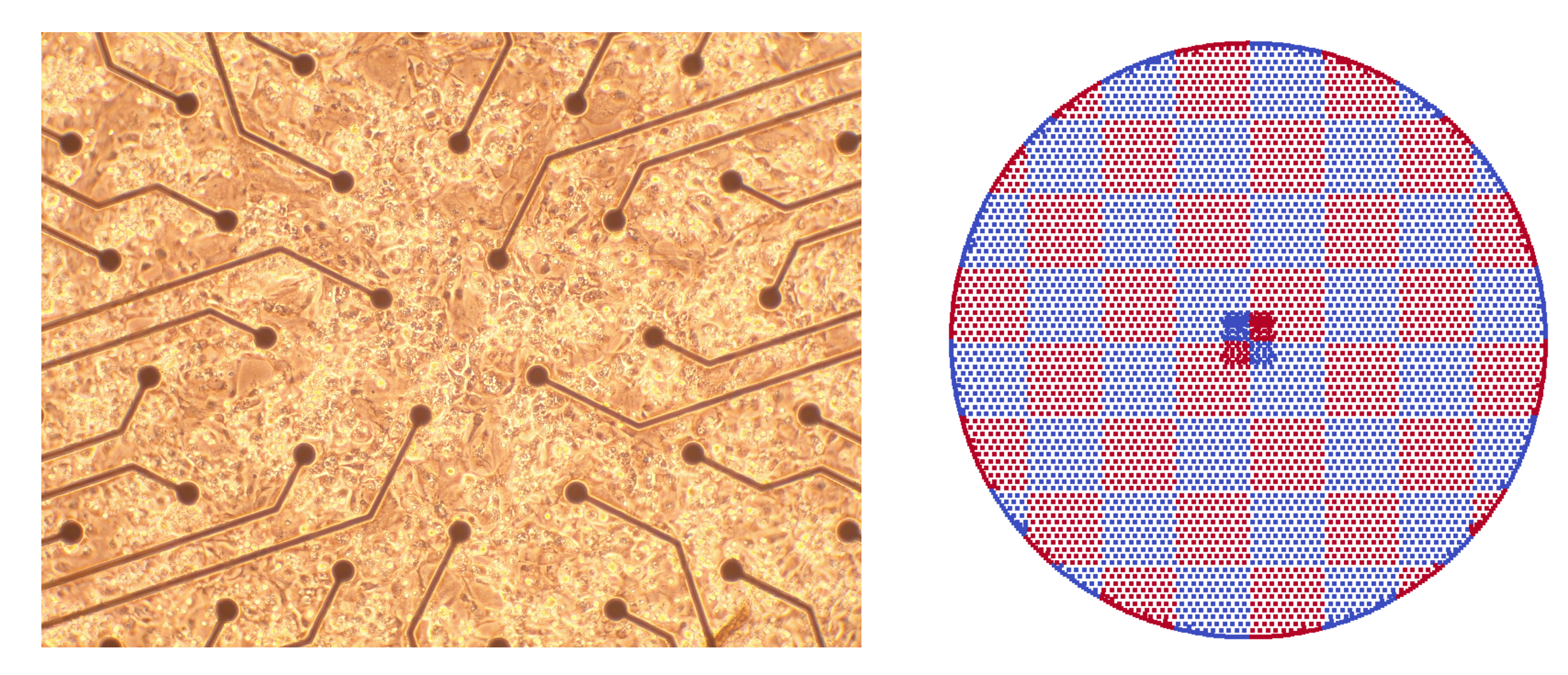

Far left: hiPSC-CM monolayer on an MEA.

Left: Nodes in an MEA mesh showing the atrial-like (red) and ventricular-like (blue) cellular electrophysiology models, In this example, the inter-node spacing is $250 \mu \mathrm{m}$.

\section{Ion Channel Block in the MEA}

The effects of ion channel blocking drugs on the simulated MEA were studied by altering the conductances of the relevant channels. The degree of block at each drug concentration (given in $\mathrm{nM}$ ) was calculated from the $\mathrm{IC}_{50}$ values (Mirams et al., 2011). Biomarkers from the simulated data were compared to experimental results (Harris et al., 2013).

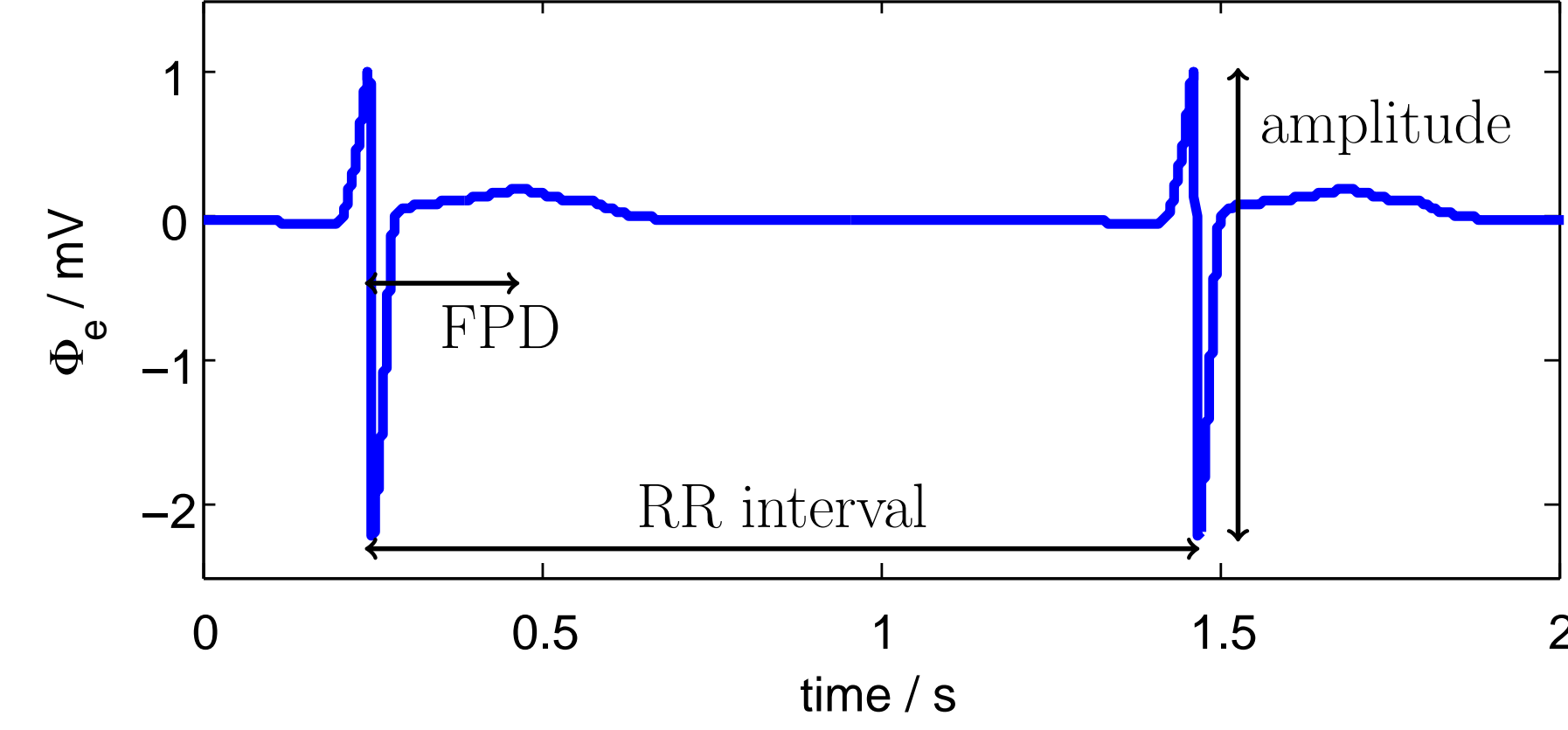

Left: Example of a typical simulated extracellular potential, labelled with the biomarkers used in this analysis. Corrected field potential duration (FPDc) was calculated from the FPD using Fridericia's formula.

Cisapride: hERG channel blocker

- The change in FPDc is larger in the simulated results than in the experimental results.

- Arrhythmias were reported in experiments at the highest cisapride concentration $(1 \mu \mathrm{M})$.

- Cells in other batches were reported to show a stronger response to similar hERG blockers than those shown here: low $I_{\mathrm{Kr}}$ in this batch of cells could have affected the results.
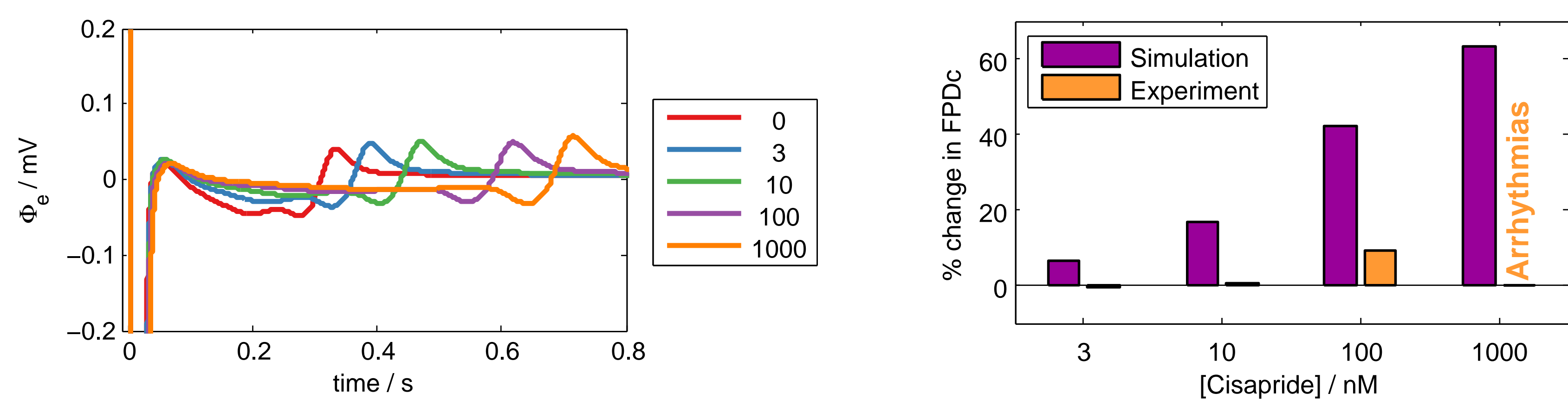

Nifedipine: $\mathrm{Ca}^{2+}$ channel blocker

- The change in FPDc agrees with the experimental results.

- The form of the potential is distorted at the highest concentration of Nifedipine.
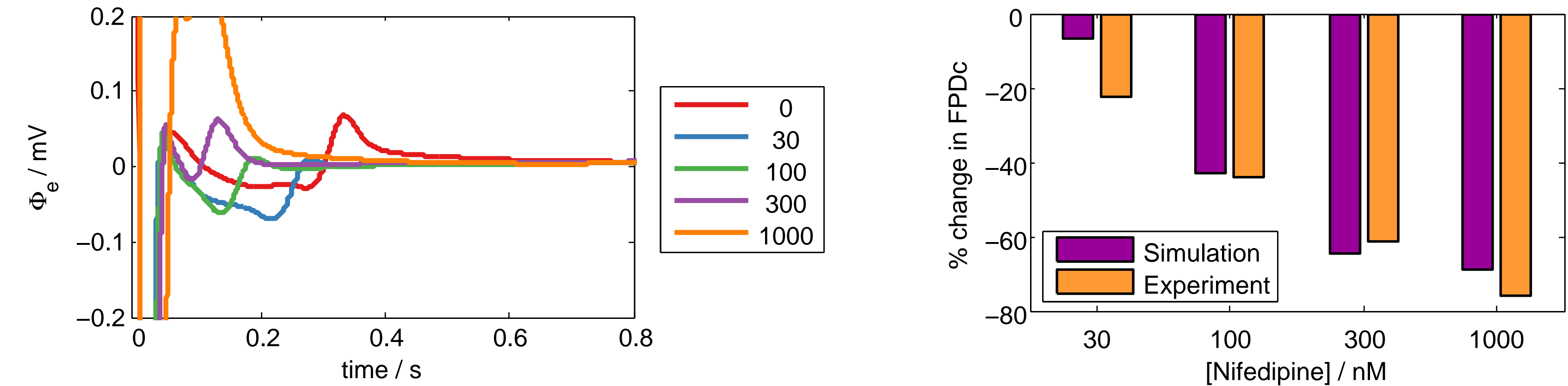

Terfenadine: mixed channel blocker

- The simulated results follow the trend of the experimental ones, with an enhanced effect.

- As Terfenadine mainly blocks hERG at lower concentrations, it is possible that differences in $I_{\mathrm{Kr}}$ in control conditions are responsible for some of the differences.

- The reduction in FPDc at high concentrations is captured well by the simulated system.
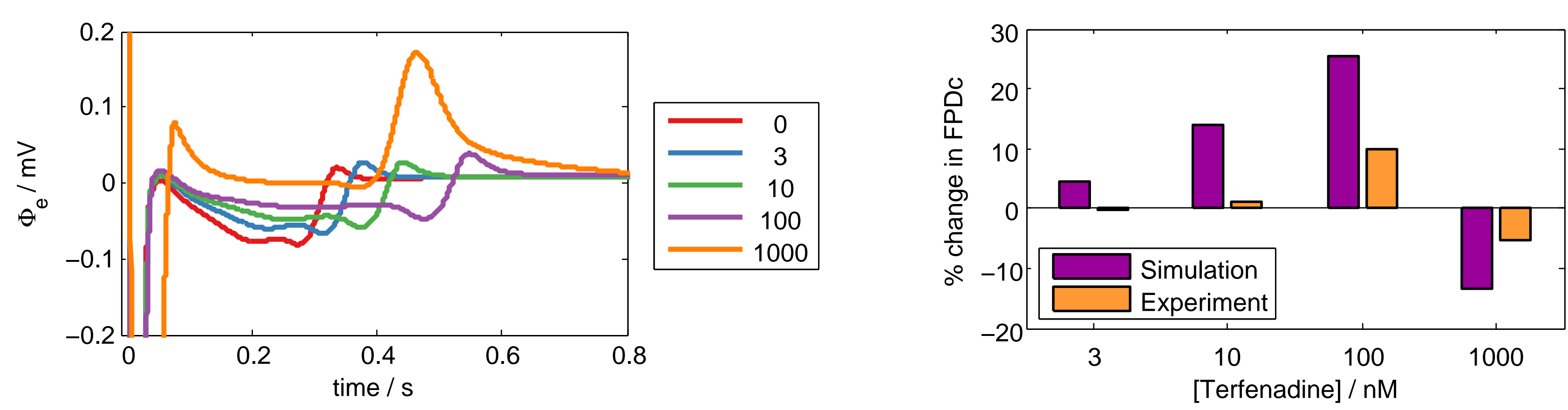

Simulations of the MEA can reproduce the observed trends in corrected field potential duration, but do not yet consistently provide quantitative predictions of drug effects.

\section{Future Work}

- Investigate alternative ways of representing regions of atrial-like and ventricular-like phenotype. This would include different sizes of atrial-like or ventricular-like regions, and the use of a mixed atrial-ventricular model for some or all of the simulated tissue.

- Repeat the simulations using $\mathrm{IC}_{50}$ values from alternative sources, such as highthroughput screening data.

- Identify aspects of the electrophysiology models which could be improved, and consider whether the models should be re-parametrised for a given batch of cells.
Harris, K., M. Aylott, Y. Cui, J. B. Louttit, N. C. McMahon, and A. Sridhar (2013). Toxicol. Sci. 134(2): 412-426.

Mirams, G. R., Y. Cui, A. Sher, M. Fink, J. Cooper, B. M. Heath, N. C. McMahon, D. J. Gavaghan and D. Noble (2011). Cardiovasc. Res. 91(1): 53-61.

Paci, M.. J. Hyttinen, K. Aalto-Setälä, and S. Severi (2013). Ann. Biomed. Eng. 41(11): 2334-2348.
If you have any questions about this poster, please ask me or get in touch via email:

louise.bowler@cs.ox.ac.uk 